\title{
Histo-Pathological Study of Endometrial Biopsies in Patients With Abnormal Uterine Bleeding
}

\author{
Sharmila Parajuli' and Binita Thapa ${ }^{2}$
}

${ }^{1}$ Department of Pathology, Kathmandu Model Hospital; ${ }^{2}$ Valley Maternity Hospital , Kathmandu, Nepal.

\section{ABSTRACT}

Introduction: Abnormal uterine bleeding is a common sign of a number of different uterine disorders ranging from dysfunctional (non organic) abnormalities or complications of pregnancy to organic lesions such as polyps, hyperplasia or carcinoma.

Methods: This is a retrospective study conducted at Hospital of 2 nd author during a period of 5 years (Jan 2008-Dec 2013). Histopathology records were retrieved and searched for cases of abnormal uterine bleeding. Relevant histopathological findings and clinical data were recorded and analyzed. The aim of the study was to determine the causes for abnormal uterine bleeding in women presenting to the hospital and to compare the histopathological findings at various age groups.

Results: The age of patients ranged from 17 to 71 years with an average of 43 years. The most common cause of uterine bleeding was found to be proliferative phase endometrium; that were 649 cases $(56.43 \%)$. Out of the pathological causes, the most common cause was found to be endometrial hyperplasia- 44 cases (3.82\%). Endometrial carcinoma was found to be more common in the elderly postmenopausal women. A total of 6 cases $(0.5 \%)$ of endometrial carcinoma were present.

Conclusion: Endometrial hyperplasias and malignancies are common in increasing age group, especially in perimenopausal and postmenopausal women. So, a thorough work-up and diagnostic endometrial biopsy is therefore mandatory without delay in these patients to rule out malignancies.

Keywords: endometrial biopsy; endometrial hyperplasia, malignancy; peri-menopausal bleeding

\section{INTRODUCTION}

Abnormal uterine bleeding is a common sign of a number of different uterine disorders ranging from dysfunctional (non organic) abnormalities or complications of pregnancy to organic lesions such as polyps, hyperplasia or carcinoma. ${ }^{1}$

A practical approach to the possible diagnosis associated with abnormal bleeding takes age into account. Pregnancy-related and dysfunctional disorders are more common in younger patients whereas atrophy and organic lesions become more frequent in older individuals. $^{2}$

Correspondence: Sharmila Parajuli; Department of Pathology, Kathmandu Model Hospital, Kathmandu, Nepal. E-mail: drsharmi@hotmail.com 
Atrophy and carcinoma occur more frequently in patients older than 60 years of age, whereas polyps and hyperplasia are more common in patients who are perimenopausal or recently postmenopausal. ${ }^{3,4}$

Ideally, the clinical history that accompanies an endometrial sample should include some description of the pattern and the amount of bleeding. ${ }^{5}$ A history of anovulation, obesity, hypertension, diabetes and exogenous estrogen use should alert the pathologist that the patient is at an increased risk for hyperplasia and adenocarcinoma. ${ }^{6}$

As the endometrium is responsive to hormones, the history of hormone use is important information. Clinical uses of steroid hormones (estrogen, progesterone or both) include oral contraceptive use, post menopausal replacement therapy and therapy for endometriosis, hyperplasia, DUB, infertility and breast carcinoma. ${ }^{7}$ Abnormal and excessive endometrial bleeding without structural pathology occurs in reproductive women of all ages but is more common in adolescent and perimenopausal women. ${ }^{8,9}$

The present study was carried out to determine the causes for abnormal uterine bleeding in women presenting in hospital to which second author is affiliated and to compare the histopathological findings at various age groups.

\section{METHODS}

This was a retrospective cross-sectional study conducted for a duration of 5 years (Jan 2008Dec 2013). The ethical approval was granted by the institutional research committee of the hospital. Verbal consent was taken from all patients for fulfilling semi-structured interview questionnaire. Demographic data including age and clinical presentation were retrieved from the histopathology records.

The criteria for selection of cases were mainly based on history and clinical examination. All patients with abnormal uterine bleeding except those with pregnancy complications were included in the study. Clinical examination was carried out as per standard protocols. Necessary radiological and laboratory investigations were done. Biopsies were taken from all the cases and the slides evaluated after processing to ascertain the cause of abnormal uterine bleeding.

Specimens were fixed in $10 \%$ formalin overnight and processed in a fully automated tissue processor. The sections were made with microtome and stained with hematoxylin and eosin stain. (H \& E stain)

\section{RESULTS}

The majority of the patients were in the age group of 40-50 years; 391 cases (34\%). The least number of cases were below the age group of 20 years- 05 cases. $(0.43 \%)$. (Table 1 )

The most common cause of uterine bleeding was found to be proliferative phase endometrium -649 cases $(56.43 \%)$. Out of the pathological causes, the most common cause

Table 1: Distribution of patients with age

\begin{tabular}{|l|c|}
\hline \multicolumn{1}{|c|}{ Age groups } & Total number of cases \\
\hline$<20$ & $05(0.43 \%)$ \\
\hline $20-30$ & $154(13.39 \%)$ \\
\hline $30-40$ & $337(29.30 \%)$ \\
\hline $40-50$ & $391(34 \%)$ \\
\hline $50-60$ & $162(14.08 \%)$ \\
\hline$>60$ & $101(8.78 \%)$ \\
\hline & $1150(100 \%)$ \\
\hline
\end{tabular}


was found to be endometrial hyperplasia; 44 cases $(3.82 \%)$._.Six cases $(0.5 \%)$ of endometrial carcinoma were diagnosed. (Table 2, Fig $1 \&$ Fig 2).

\section{DISCUSSION}

The commonest age group of our study was between 40-50 years (391 cases-34\%). This study well correlated with other similar studies conducted in Pakistan by Yusuf NW and Luqman $M$ which showed the highest peak in the same age group in the frequency of $38.06 \%$

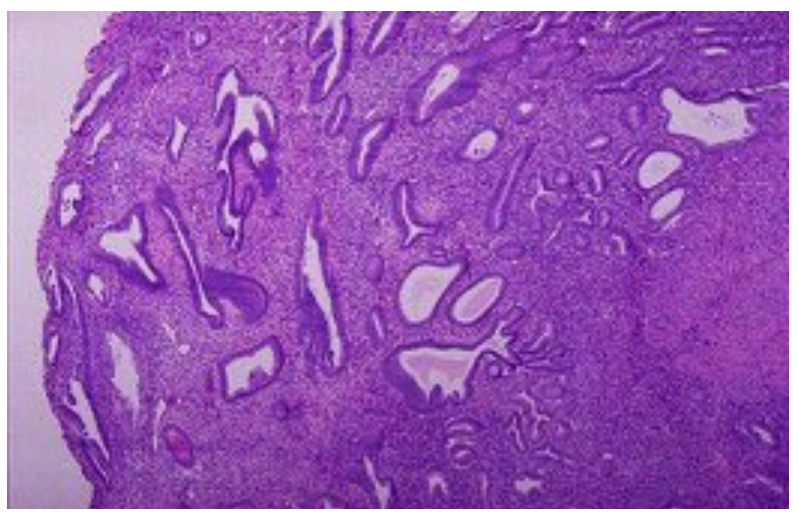

Fig 1: Simple cystic hyperplasia without atypia (H\&E stain $\mathrm{x} 10)$ and $30 \% .10,11$ Vakiani et al, in their study found the mean age group of the patient as 40 years. ${ }^{12}$

The most common pathological histopathological diagnosis of abnormal uterine bleeding was found to be endometrial hyperplasia- 44 cases $(3.82 \%)$. This compared favorably with $3.8 \%$ in other studies conducted at the Islamic international medical college. ${ }^{10,}$ 11

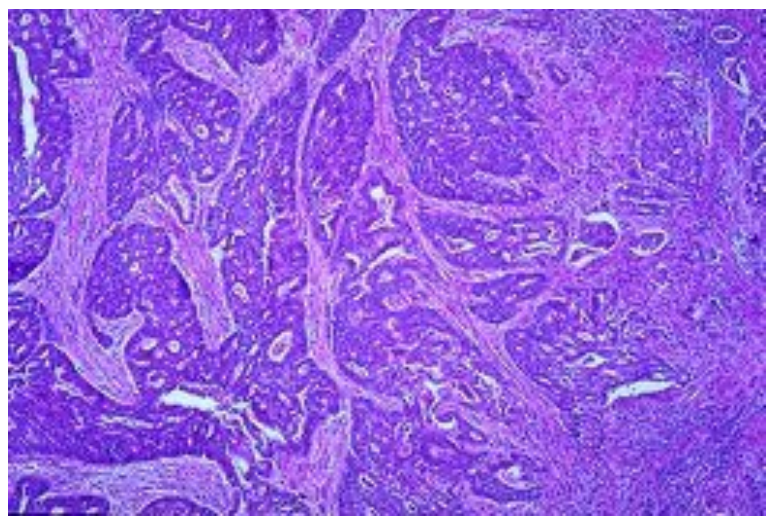

Fig1: Adenocarcinoma-endometrium showing neoplastic glands(H\&E X 10)

Table 1: Histo-pathological diagnosis of patients with age.

\begin{tabular}{|c|c|c|c|c|c|c|c|}
\hline Findings & $<20$ & 20-30 & $30-40$ & $40-50$ & $50-60$ & $>60$ & Total \\
\hline Atrophic endometrium & 0 & 0 & 0 & 7 & 113 & 97 & $217(18.86 \%)$ \\
\hline Proliferative phase & 3 & 110 & 229 & 296 & 11 & 0 & $649(56.43 \%)$ \\
\hline Secretory phase & 2 & 44 & 79 & 27 & 0 & 0 & $152(13.21 \%)$ \\
\hline Endometrial hyperplasia & 0 & 0 & 4 & 26 & 14 & 0 & $44(3.82 \%)$ \\
\hline Endometrial polyp & 0 & 0 & 1 & 3 & 2 & 1 & $7(0.6 \%)$ \\
\hline $\begin{array}{l}\text { Endometritis } \\
\text { (Chronic) }\end{array}$ & 0 & 0 & 0 & 1 & 4 & 1 & $6(0.5 \%)$ \\
\hline Pill endometrium & 0 & 0 & 0 & 24 & 11 & 0 & $35(3.04 \%)$ \\
\hline $\begin{array}{l}\text { Disordered proliferative } \\
\text { phase endometrium }\end{array}$ & 0 & 0 & 23 & 7 & 4 & 0 & $34(2.95 \%)$ \\
\hline Endometrial carcinoma & 0 & 0 & 1 & 0 & 3 & 2 & $6(0.5 \%)$ \\
\hline Total & 5 & 154 & 337 & 391 & 162 & 101 & 1150 \\
\hline
\end{tabular}


Endometrial hyperplasia was the most common histopathological diagnosis in our study accounting for a total of 44 cases $(3.8 \%)$. This compared favorably with $3 \%$ in a study conducted by Yusuf NW and his colleagues at the Islamic international medical college. 10 Out of the 44 patients diagnosed as endometrial hyperplasia in our study, all were diagnosed as simple cystic hyperplasia without atypia- 39 cases (98\%) except for 2 cases diagnosed as simple hyperplasia with atypia and 3 cases diagnosed as complex atypical hyperplasia. A slightly decreased incidence of simple cystic hyperplasia was reported by Vakiani et al ${ }^{12}-71.67 \%$. Yusuf NW et al and Luqman $\mathrm{M}$ et al also showed the frequency of simple cystic hyperplasia in the range of $14 \%$ and $17.9 \%$ respectively. 10,11

The incidence of atypical and adenomatous hyperplasia however increased in these studies. 12 This is explained by the fact that the incidence of endometrial carcinoma was also high in these studies. The more severe the hyperplasia, the more likely it is to be followed by endometrial carcinoma. In the case of simple hyperplasia, the risk is very small. Conversely, the incidence of carcinoma in women with complex and atypical hyperplasia has been in the neighborhood of $15-30 \% .{ }^{13}$

The lower frequency of atypical and adenomatous hyperplasia in our study can be explained by the fact that Nepalese women practice early childbearing and multiparity leading to a much longer resting (inactive state) of the endometrium. Further there is also a reduced incidence of carcinoma in this study. $(0.5 \%)$

The normal physiological pattern of proliferative phase endometrium -649 $(56.43 \%)$ was slightly higher as compared to
$48 \%$ reported by $\operatorname{Nadoss}^{14}$. Other studies quoted a marked decreased incidence as $24 \%$, $25 \%$ and $26 \% 10,11$.

The incidence of atrophic endometrium in our study was 217 (18.86\%). Postmenopausally, DUB is frequently associated with an atrophic endometrium- an unprepared endometrium resulting from inadequate secretion of estrogen. ${ }^{9}$

The incidence of chronic endometritis was less- $0.5 \%$ as compared to $24 \%$ in other studies. 10,11 Chronic endometritis characterized by an infiltrate of lymphocytes and plasma cells usually follows pregnancy or abortion and use of intracontraceptive device (IUCD) ${ }^{9}$

Frequency of endometrial polyp was slightly lesser- $0.6 \%$ as compared to $3.2 \%$ in a study conducted in Pakistan. ${ }^{10} 1$ case of endometrial polyp was present in a patient who was previously diagnosed as breast carcinoma and who was receiving Tamoxifen for treatment of breast carcinoma. Tamoxifen is a non-steroidal drug that has a therapeutic anti-oestrogen effect on the breast and an estrogenic effect on the endometrium of post-menopausal women. Patients with breast cancer or prolonged tamoxifen therapy are reported to have increased risk of developing endometrial polyps, hyperplasia and carcinoma. ${ }^{15}$

Use of hormonal pills also predispose to a condition known as pill endometrium, especially use of progestational agents. Pill pattern endometrium was almost the same- 3\% as that as $2.8 \%$. ${ }^{10}$ Oral contraceptive pills and hormonal use predispose to endometrial hyperplasia.

As the women get old, the incidence of structural abnormality including malignancy increases. ${ }^{16}$ A total of $6(0.5 \%)$ cases in our study had endometrial carcinomas. Out of the 6 
cases, 2 cases were diagnosed as adenosquamous carcinoma and 1 case was diagnosed as Adenoacanthoma. There was increased risk to develop malignancy in patients with diabetes and hypertension with a history of nulliparity. In a study done by Feng Y, malignancies of the genital tract accounted for $30-50 \%$ of cases of bleeding in the postmenopausal period and $15 \%$ of all such cases had endometrial carcinoma. ${ }^{17}$ A study conducted by Bayer and De Cherney in the department of Gynae in the cancer institute, Japan showed an incidence of endometrial carcinoma as $2.8 \%{ }^{18}$

The incidence of endometrial carcinoma is comparable to other studies except a study conducted in Chinese population which showed a high incidence of $50 \% .{ }^{17}$

The lower incidence of endometrial carcinoma in Nepalese women may be due to the practice of early childbearing and multiparity leading to a much longer resting (inactive state) during pregnancy. ${ }^{19}$

\section{CONCLUSIONS}

Endometrial hyperplasia and malignancies are common in increasing age group, especially in perimenopausal and postmenopausal women. So, a thorough work-up and diagnostic endometrial biopsy is therefore mandatory without delay in these patients to rule out malignancies.

\section{REFERENCES}

1. Galle PC, McRae MA; Abnormal Uterine bleeding. Finding and treating the cause: Post graduate med 1993; 93:73-81

2. Povey WC. Abnormal uterine bleeding at puberty and climacteric. Clin Obstetric. Gynaecology. 1970; 13: 474-488

3. Archer DF, McIntyre- Seltman k, Wilborn WW, Dowling EA, Cone F, Creasy GW, et al. Endometrial morphology in asymptomatic postmenopausal women. Am J obstetric Gynaecol. 1991; 165: 317-322

4. Gredmark T, Kvint S, Havel G, Mattsson LA. Histopathological findings in women with postmenopausal bleeding, Br J Obstet Gynaecol 1995;102: 133-136

5. Merrill JA. The interpretation of endometrial biopsies, Clin obstet Gynae 1991; 34: 211-221

6. Schindler AE, Schmidt G, Post-menopausal bleeding: A study of more than 1000 cases; Maturitas 1980; 2: 269-274

7. Aksel S, Jones GS. Etiology and treatment of dysfunctional uterine bleeding. J obstetr. Gynaecol 1974; 44:1-13

8. Sharma JB. DUB, Obs and Gynae Today, 2000; 5(11): 20-25

9. Davey DA. DUB In: Whit field CR, ed, Dewhurtst's Textbook of obstetric and gynaecology for Postgraduates, Glasgow, Blackwell Science 1997: 590-6084.

10. Yusuf NW, Nadeem R, Yusuf AW, Rahman R. Dysfunctional uterine bleeding. A Retrospective clinicopathological study over 2 years. Pak J. Obstet Gynaecol 1996; 9:27-30.

11. Luqman M, Bukhari L. Abnormal/ Excessive uterine haemorrhage - A histopathological study. Pakistan J Pathol 1998; 9:20-24.

12. Vakiani et al- Clin Exp. Obstetrics and Gynaecology 1996; 23: 236-239.

13. Fox $\mathrm{H}$, Buckley $\mathrm{CH}$. The endometrial hyperplasias and their relationship to endometrial neoplasia. Histopathology. 1982; 6: 493-510.

14. Nadoss BR. Dysfunctional uterine bleeding. Relation of endometrial histology 
to outcome. Am J Obsted Gynaecol

1971;109:103-7.

15. Anonymous, $\mathrm{Ca}$ of the endometrium, In: Padubidri VG, Daftary SN, eds. Shaw's textbook of Gynaecology, 12 $2^{\text {th }}$ ed; New Delhi, Curchill Livington, 1999: 302-309.

16. Sharma JB. Dysfunctional Uterine bleeding( DUB). Obstetrics and Gynecology Today, 2000; 5(11): 20-25.

17. Feng Y; Clinical analysis in 56 cases of $\mathrm{P} / \mathrm{V}$ bleeding, HeBei Medical Journal, 1989; 12:178-181.

18. Bayer SR, De Cherney AH. Clinical manifestations and treatment of Dysfunctional uterine bleeding. JAMA, 1993; 269: 1823-1828.

19. Dangal G. A study of endometrium of Patients with abnormal uterine bleeding at Chitwan Valley, KUMJ, 2003, Vol 1, No 2: 110-112. 